\title{
3-(2-萗基)-1-苯基-吡唑-4-甲醛腙衍生物的简便合成与表征
}

\author{
阿布拉江・克依木* 王立举 \\ (新疆大学化学化工学院 新疆石油天然气精细化工教育部\&自治区重点实验室 乌鲁木齐 830046)
}

\begin{abstract}
摘要 为寻找具有生物活性的先导化合物, 以 3-(2-菜基)-1-苯基-吡唑-4-甲醛为起始原料, 在超声波辐射下经串联反应 和 “一锅法合成” 两种途径制备了一系列新型的 1-(3- $\beta$-菜基-1-苯基吡唑-4-亚甲基)-2-(4-芳基噻唑-2-基)-腙及其衍生物. 与加热实验相比, 在超声波辐射下可得到较好的产率. 利用 ${ }^{1} \mathrm{H} N \mathrm{NM}, \mathrm{MS}$, IR 谱和元素分析对产物进行了结构表征. 初 步的生物活性测试结果表明, 化合物 $\mathbf{4 b}$ 和 $\mathbf{4 d}$ 对 $\mathrm{H}_{2} \mathrm{O}_{2}$ 诱导的 PC12 细胞损伤具有明显的保护作用.

关键词 吡唑; 噻唑; 腙; 合成; 超声辐射; 一锅反应
\end{abstract}

\section{Convenient Synthesis and Characterization of Hydrazone Derivatives of 3-(2-Naphthyl)-1-phenyl-pyrazole-4-carbaldehyde}

\author{
Ablajan, Keyume* Wang, Liju \\ (Key Laboratory of Oil \& Gas Fine Chemical, Ministry of Education \& Xinjiang Uyghur Autonomous Region, \\ College of Chemistry and Chemical Engineering, Xinjiang University, Urumqi 830040)
}

\begin{abstract}
In order to obtain novel hydrazone derivatives containing pyrazole and thiazole rings which possess certain biological activity, a series of 1-(3- $\beta$-naphthyl-1-phenylpyrazole-4-methylene)-2-(4-arylthiazol-2-yl)hydrazones were synthesized via two different methods including multi step reactions and one-pot synthetic route using 3- $\beta$-naphthyl-1-phenyl-pyrazole-4-carbaldehyde as a starting intermediate. The products were obtained in good yield under ultrasonic irradiation condition rather than heating. The structures of products were characterized by ${ }^{1} \mathrm{H}$ NMR, MS and elemental analysis. The preliminary bioassay showed that compounds $\mathbf{4 b}$ and $\mathbf{4 d}$ possess obvious protective effects on the $\mathrm{PC} 12$ cells injury induced by $\mathrm{H}_{2} \mathrm{O}_{2}$.

Keywords pyrazole; thiazole; hydrazone; synthesis; ultrasonic irradiation; one-pot reaction
\end{abstract}

吡唑类化合物是一类具有广泛生物活性的杂环化 合物, 在医药和农药方面具有十分重要的应用前景, 并 且已有许多吡唑类农药相继商品化. 许多含吡唑基团的 化合物除了具有良好的除草、杀虫、杀菌等 ${ }^{[1,2]}$ 活性外, 还具有抗菌、抗炎、抗结核、抗寄生等 ${ }^{[3 \sim 5]}$ 生物活性. 腙 类化合物是一类合成杂环化合物的重要中间体, 由于其 分子结构中含有亚胺结构 $(\mathrm{NHN}=\mathrm{C})$, 可以衍生出很多 具有较高生物活性的化合物. 研究表明, 腙类化合物可 以表现出抗病毒、抗肿瘤和杀菌等活性, 可以抑制体内 诸多生理生化过程 ${ }^{[6 \sim 8]}$. 噻唑环和腙类结构片段作为重 要的结构单元或中间体也同时出现在许多医药和农药 之中, 许多调查表明, 它们在高效农药创制、新型发光 材料研究等领域中发挥出越来越重要的作用而倍受广 泛关注 ${ }^{[9 \sim 12]}$. 一般来讲, 1-芳基-2-(4-芳基噻唑-2-基)-腙
类通常经两步合成制得. 首先将氨基硫腿与芳香醛或酮 反应生成芳香醛(或酮)缩氨基硫脲, 然后其与 $\omega$-溴代芳 基乙酮发生缩合环化反应生成含噻唑环的腙类化合 物 ${ }^{[13,14]}$. 另外, 通过多组分一锅法也可以合成多取代苯 基噻唑腙类化合物 ${ }^{[15]}$.

为了寻找具有潜在生物活性的先导化合物并对此 类杂环类化合物的合成提供简便及有效的方法与途径, 我们首先将菜乙酮苯腙与 Vilsmeier-Haak 试剂反应制得 新颖的中间体 3- $\beta$-芸基-1-苯基吡唑-4-甲醛(2). 再将化 合物 2 作为起始原料, 设计合成了一系列新型的 1-(3- $\beta$-菜基-1-苯基吡唑-4-亚甲基)-2-(4-芳基噻唑-2-基)腙 $(\mathbf{4 a} \sim \mathbf{4 g})$. 另外，在串联反应的基础上，设计实现了以 三组分一锅法合成目标产物. 产物结构经 ${ }^{1} \mathrm{H}$ NMR, MS 谱和元素分析进行了确定及表征. 合成路线见 Scheme 1.

*E-mail: ablajan209@hotmail.com

Received July 31, 2012; revised August 15, 2012; published online August 22, 2012.

Project supported by the Natural Science Foundation of Xinjiang Uyghur Autonomous Region (No. 2009211A02).

新疆维吾尔自治区自然科学基金(No. 2009211A02)资助项目. 


\section{1 结果与讨论}

\section{1 合成}

杂环基醛腙类化合物的合成方法比较成熟，一般采 用常规回流法, 回流时间一般为 $1 \mathrm{~h}$ 以上, 而超声辐射 已成为合成噻唑类化合物的重要辅助技术. 本研究中, 我们通过方法 A 分别采用常规回流法和超声波辐射反 应法合成了目标化合物, 得知经超声波辐射反应所得到 的产率比常规回流法约大 $10 \%$. 这说明, 在加热条件下 反应物可能产生一些副产物, 而超声波辐射反应可以作 为温和的手段，能提高反应产率. 此外，因为芳基乙酮 分子中取代基不同，反应所需要的时间也不同. 虽然对 于化合物 $4 \mathrm{~g}$ 在加热回流下反应所需要的时间最短, 5 min 内就结束, 但其反应产率比超声波辐射下要低一些. 其余产物生成所需要的时间稍长. 由此可知, $3-\beta$-萗基1-苯基吡唑-4-甲醛缩氨基硫脲与 $\omega$-溴代芳基乙酮在超 声波辐射下反应可以得到产率较好的产物 $\mathbf{4 a} \sim \mathbf{4 g}$. 相
关数据见表 1 .

基于多组分一锅法合成具有降低能耗、节省时间、 减少分离和纯化步骤、提高产品产率和环境友好等特点, 已成为合成许多杂环类化合物的重要方法. 我们在方法 A 的研究结果基础上, 又设计了合成方法 B. 在超声 波辐射下通过三组分一锅法合成了目标化合物 $\mathbf{4 a} \sim \mathbf{4 g}$, 也取得了良好的结果. 虽然与方法 $\mathrm{A}$ 相比, 个别化合 物反应时间稍长, 但是方法 $\mathrm{B}$ 操作相对简单, 并且产 率相对较高. 因此, 其具有较好的优势. 反应时间及产 率如表 1 所示. 另外, 目标产物生成的可能机理如 Scheme 2 所示, 首先氨基硫脲分子上氨基与醛羰基缩 合脱去一分子水，与此同时氨基硫腿分子中的颈基进攻 $\omega$-澳代芳基乙酮的亚甲基，脱去一分子 $\mathrm{HBr}$ ，生成中间 体 5A. 随后中间体 5A 中的羰基先质子化, 分子内亚胺 基亲核进攻羰基碳原子形成新的羟基，然后羟基经质子 化脱去一分子 $\mathrm{H}_{2} \mathrm{O}$ 生成目标产物 4.

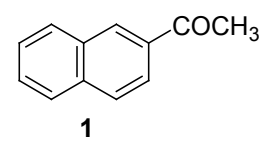

(a) $\mathrm{PhNHNH}_{2}$

(b) DMF/POCl 3

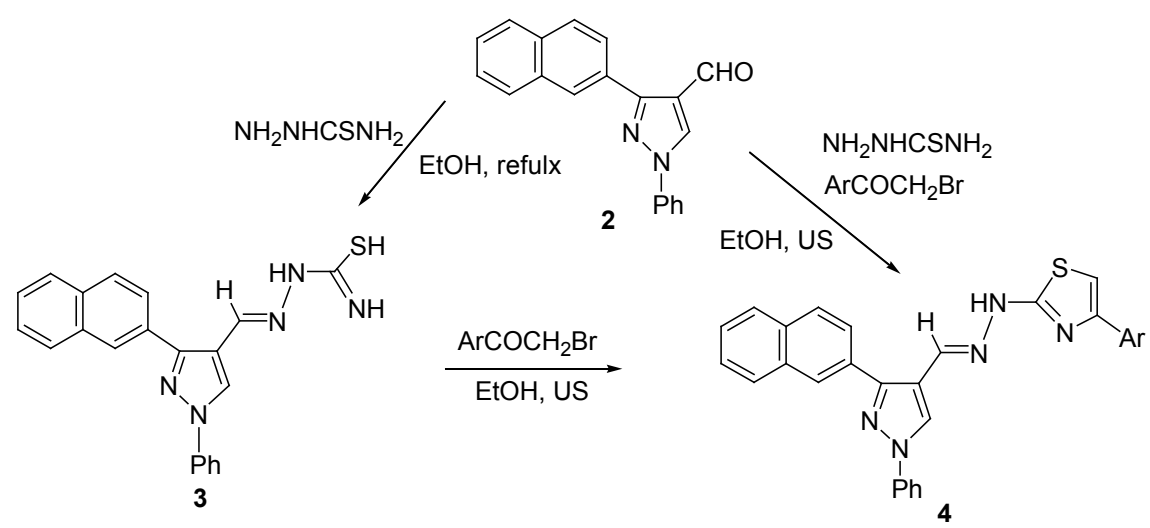

$\mathrm{Ar}=\mathrm{C}_{6} \mathrm{H}_{5}(\mathbf{4 a}), 4-\mathrm{H}_{3} \mathrm{CC}_{6} \mathrm{H}_{4}$ (4b), 4- $\mathrm{H}_{3} \mathrm{COC}_{6} \mathrm{H}_{4}$ (4c), 4- $\mathrm{ClC}_{6} \mathrm{H}_{4}$ (4d), 4- $\mathrm{O}_{2} \mathrm{NC}_{6} \mathrm{H}_{4}$ (4e), 4-BrC $\mathrm{H}_{4}$ (4f), 2-Naphthyl (4g)

Scheme 1

表 1 不同方法所得到产物的时间和产率

Table 1 Time and yield for the product obtained via different method

\begin{tabular}{cclccc}
\hline Entry & Product & \multicolumn{1}{c}{$\mathrm{Ar}$} & Time $^{a}(\mathrm{~min}) / \mathrm{Yield}^{a}(\%)$ & Time $^{b}(\mathrm{~min}) / \mathrm{Yield}^{b}(\%)$ & Time $^{c}\left(\mathrm{~min}^{2} / \mathrm{Yield}^{c}(\%)\right.$ \\
\hline 1 & $\mathbf{4 a}$ & $\mathrm{C}_{6} \mathrm{H}_{5}$ & $17 / 65$ & $60 / 74$ & $50 / 70$ \\
2 & $\mathbf{4 b}$ & $4-\mathrm{H}_{3} \mathrm{CC}_{6} \mathrm{H}_{4}$ & $14 / 73$ & $45 / 79$ & $60 / 70$ \\
3 & $\mathbf{4 c}$ & $4-\mathrm{H}_{3} \mathrm{COC}_{6} \mathrm{H}_{4}$ & $12 / 76$ & $45 / 83$ & $60 / 80$ \\
4 & $\mathbf{4 d}$ & $4-\mathrm{ClC}_{6} \mathrm{H}_{4}$ & $8 / 69$ & $40 / 87$ & $45 / 75$ \\
5 & $\mathbf{4 e}$ & $4-\mathrm{O}_{2} \mathrm{NC}_{6} \mathrm{H}_{4}$ & $30 / 82$ & $60 / 89$ & $60 / 85$ \\
6 & $\mathbf{4 f}$ & $4-\mathrm{BrC}_{6} \mathrm{H}_{4}$ & $6 / 61$ & $30 / 72$ & $45 / 70$ \\
7 & $\mathbf{4 g}$ & $2-\mathrm{Naphthyl}$ & $3 / 76$ & $20 / 85$ & $30 / 80$ \\
\hline
\end{tabular}

Method A: ${ }^{a}$ refluxing condition; ${ }^{b}$ ultrasonic irradiation; Method $\mathrm{B}:{ }^{c}$ one-pot reaction under ultrasonic irradiation. 


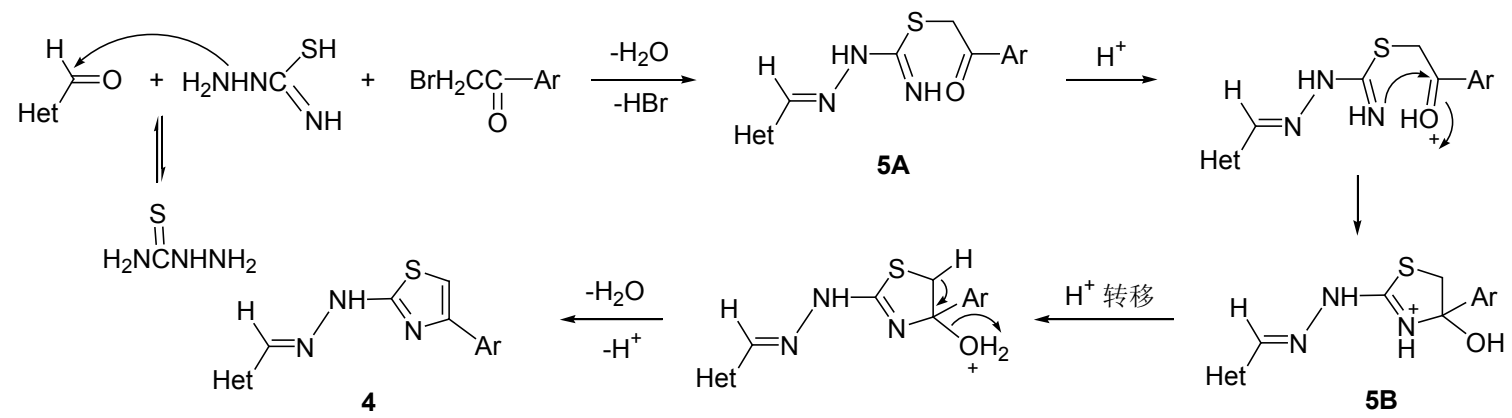

Het: 3-(2-Naphthyl)-1-phenyl-pyrazol-4-yl

Scheme 2

\section{2 波谱解析}

目标化合物 $\mathbf{4 a} \sim \mathbf{4 g}$ 的质谱中均出现 $[\mathrm{M}+\mathrm{H}]^{+}$和 $[\mathrm{M}+\mathrm{Na}]^{+}$峰, 可以推断化合物的准确分子量. 当分子中 含有氯或澳时, 都出现了 $[\mathrm{M}+2+\mathrm{H}]^{+}$或 $[\mathrm{M}+2+\mathrm{Na}]^{+}$同 位素峰. 在 ${ }^{1} \mathrm{H}$ NMR 谱中, $\delta 11.37 \sim 12.15$ 出现的一个单 峰为 $\mathrm{N}-\mathrm{H}$ 的质子吸收峰; $\delta \quad 6.95 \sim 8.40$ 处为萗环和芳 环-H 的质子吸收峰; $\delta 8.06 \sim 8.14$ 范围内出现的单峰为 $\mathrm{N}=\mathrm{C}-\mathrm{H}$ 上的质子信号; 由于受到吡唑环的强共轭效 应影响, 吡唑环中 $\mathrm{H}$ 的吸收峰出现在 $\delta 8.55 \sim 9.25$ 范 围内. 在红外光谱中, 发现 $3350 \mathrm{~cm}^{-1}$ 左右出现 $\mathrm{N}-\mathrm{H}$ 的 伸缩振动吸收峰, 在 $1630 \sim 1490 \mathrm{~cm}^{-1}$ 左右四组峰为 $\mathrm{C}=\mathrm{N}$ 和 $\mathrm{C}=\mathrm{C}$ 吸收峰以及芳环和吡唑环的骨架振动吸 收峰.

\section{3 生物活性}

为了篮选具有生物活性的先导化合物, 我们研究了 目标化合物对 $\mathrm{H}_{2} \mathrm{O}_{2}$ 诱导 PC12 细胞损伤的影响. 结果 表明, 化合物 $4 \mathrm{~b}$ 和 $4 \mathrm{~d}$ 处理组, 细胞形态明显改善, MTT 吸光值明显增加, 这说明化合物 $4 \boldsymbol{b}$ 和 $4 \boldsymbol{d}$ 能减轻 $\mathrm{H}_{2} \mathrm{O}_{2}$ 氧化损伤所致的 PC12 神经细胞的死亡, 对 $\mathrm{H}_{2} \mathrm{O}_{2}$ 诱导的 PC12 细胞损伤具有明显的保护作用. MTT 法检测结果: 细胞存活率分别为 $4 \mathbf{a}(30 \%), \mathbf{4 b}(58 \%), \mathbf{4 c}(42 \%), \mathbf{4 d}$ (62\%), 4e (46\%), 4f (39\%), 4g (34\%).

\section{2 结论}

本文报道了经串联反应和三组分一锅法反应两种 途径设计合成一系列新型 1-(3- $\beta$-䒺基-1-苯基吡唑-4-亚 甲基)-2-(4-芳基噻唑-2-基)-腙及其衍生物的研究. 结果 表明, 在超声波辐射下经三组分一锅法合成目标化合 物, 操作相对简单, 产率相对较高, 较常规回流法合成 目标化合物更具有优势.

\section{3 实验部分}

\section{1 仪器与试剂}

核磁共振仪: INOVA-400(内标 TMS, 溶剂 DMSO- $\left.d_{6}\right)$; 熔点仪: 日本 Yanaco MP-S3 (温度计经校正); 质谱 仪: HP 1100 LC-MS (ESI); 元素分析用 Perkin Elmer $2400 \mathrm{CHN}$ 型仪. 所用试剂均为分析纯，除特殊说明外 未经处理直接使用; 三氯氧磷经重蒸处理; DMF 经干 燥处理; 菜乙酮苯腙按文献[16]方法合成; $\omega$-溴代芳基 乙酮按文献[17]方法合成.

\section{2 实验方法}

\subsubsection{3- $\beta$-䒬基-1-苯基吡唑-4-甲醛(2)的合成}

参照文献[18]方法, 在冷却 $\left(0{ }^{\circ} \mathrm{C}\right)$ 及摚拌下, 向无水 $0.03 \mathrm{~mol} \mathrm{DMF}$ 中滴加 $0.09 \mathrm{~mol} \mathrm{POCl}_{3}$, 约为 $30 \mathrm{~min}$ 滴完. 滴完后继续摚拌 $30 \mathrm{~min}$, 就制成 Vilsmeier-Haak 试剂. 在冷却及剧烈搅拌下, 将事先制得到的荎乙酮苯腙的 $10 \mathrm{~mL}$ 无水 DMF 溶液在 $5 \mathrm{~min}$ 内滴入 Vilsmeier-Haak 试剂中, 在室温下继续搅拌 $30 \mathrm{~min}$, 水浴上回流 $6 \mathrm{~h}$, 冷 却至室温后倒入 $200 \mathrm{~mL}$ 冰水中, 并冷却至 $0{ }^{\circ} \mathrm{C}$. 向其中 加入 $2 \mathrm{~mol} / \mathrm{L} \mathrm{NaOH}$ 溶液, 调 $\mathrm{pH}$ 值至中性. 过滤, 放干 后, 用乙醇重结晶, 得 3- $\beta$-荎基-1-苯基吡坐-4-甲醛(2). 白色晶体, 产率 $85 \%$. m.p. $147 \sim 149{ }^{\circ} \mathrm{C} ;{ }^{1} \mathrm{H}$ NMR $\left(\mathrm{CDCl}_{3}, 400 \mathrm{MHz}\right) \delta: 10.15(\mathrm{~s}, 1 \mathrm{H}, \mathrm{CHO}), 8.59(\mathrm{~s}, 1 \mathrm{H}$, Pyrazole-H), 8.33 (s, 1H, Naphthyl-H), 7.84 (d, $J=8.8 \mathrm{~Hz}$, 1H, Naphthyl-H), 7.53 (d, $J=8.8 \mathrm{~Hz}, 1 \mathrm{H}$, Naphthyl-H), $7.40 \sim 7.80$ (m, 9H, Naphthyl-H+ArH). Anal. calcd for $\mathrm{C}_{20} \mathrm{H}_{14} \mathrm{~N}_{2} \mathrm{O}$ : C 80.54, H 4.76, N 9.41; found $\mathrm{C} 80.52, \mathrm{H}$ $4.73, \mathrm{~N} 9.39$.

3.2.2 3- $\beta$ - 菜基-1-苯基吡唑-4-甲醛缩氨基硫腿 (3)的 合成

将 $0.01 \mathrm{~mol}$ 化合物 $\mathbf{2}$ 和 $0.01 \mathrm{~mol}$ 氨基硫脲在 $30 \mathrm{~mL}$ 无水乙醇和 $2 \mathrm{~mL}$ 冰乙酸溶液中回流 $10 \mathrm{~h}$, 冷却后析出 白色固体, 用 DMF-EtOH 重结晶得 3- $\beta$-萗基-1-苯基吡 唑-4-甲醛缩氨基硫脲(3). 白色固体，产率 83\%. m.p. $226 \sim 228{ }^{\circ} \mathrm{C} ;{ }^{1} \mathrm{H}$ NMR (DMSO- $d_{6}, 400 \mathrm{MHz}$ ) $\delta: 11.37$ (s, $1 \mathrm{H}, \mathrm{NH}), 9.25(\mathrm{~s}, \quad 1 \mathrm{H}$, Pyrazole-H), $8.37(\mathrm{~s}, 1 \mathrm{H}$, Naphthyl-H), 8.28 (s, 1H, C=NH), $8.21(\mathrm{~s}, 1 \mathrm{H}, \mathrm{N}=\mathrm{CH})$, 7.84 (s, 1H, CSH), $7.39 \sim 8.07$ (m, 11H, Naphthyl-H+ 
ArH); ESI-MS m/z (\%): $394\left(\mathrm{M}+\mathrm{Na}^{+}, 86\right), 372\left(\mathrm{M}+\mathrm{H}^{+}\right.$, 10); IR (KBr) v: 3269, $3130\left(\mathrm{NH}_{2}, \mathrm{NH}\right), 2975(\mathrm{Ar}-\mathrm{H})$, 1628, 1597, 1546, $1501(\mathrm{C}=\mathrm{C}, \mathrm{C}=\mathrm{N}), 1226(\mathrm{C}-\mathrm{N}), 831$ $(\mathrm{C}=\mathrm{S}) \mathrm{cm}^{-1}$. Anal. calcd. for $\mathrm{C}_{20} \mathrm{H}_{14} \mathrm{~N}_{2} \mathrm{O}: \mathrm{C} 67.93, \mathrm{H} 4.65$, N 18.87; found C 67.90, H 4.61, N 18.85.

3.2.3 超声波辐射下 1-(3- $\beta$ - 䒬基-1-苯基吡唑-4-亚甲 基)-2-(4-芳基噻唑-2-基)-腙( $4 \mathrm{a} \sim 4 \mathrm{~g}$ )的合成

将 $1 \mathrm{mmol} 3$ - $\beta$-萘基-1-苯基吡唑-4-甲醛缩氨基硫艮 (3)加热溶于 $25 \mathrm{~mL}$ 无水乙醇中, 然后加人 $1 \mathrm{mmol} \omega$-溴 代芳基乙酮, 混合物在超声波辐射下反应 $20 \sim 60 \mathrm{~min}$ 后析出固体. 用 TLC [ $V$ (甲醇) $: V($ 氯仿 $)=1: 3$ ] 跟踪反 应. 反应结束, 滴加稀 $\mathrm{NaOH}$ 溶液中和, 过滤, 用乙醇 洗涤产物. 用 DMF-EtOH 重结晶, 得 1-(3- $\beta$-菱基-1-苯 基吡唑-4-亚甲基)-2-(4-芳基噻唑-2-基)-腙类化合物 $\mathbf{4 a} \sim \mathbf{4 g}$.

3.2.4 常规加热回流合成 1-(3- $\beta$-菜基-1-苯基吡唑-4亚甲基)-2-(4-芳基噻唑-2-基)-腙 $(\mathbf{4 a} \sim 4 \mathrm{~g}$ ) 的合成

将 $1 \mathrm{mmol} 3$ - $\beta$-荎基-1-苯基吡唑-4-甲醛缩氨基硫艮 (3)和 $1 \mathrm{mmol} \omega$-溴代芳基乙酮溶于 $25 \mathrm{~mL}$ 无水乙醇中, 加热回流反应 3 30 min, 反应过程中用 TLC [ $V$ (甲 醇) $: V($ 氯仿 $)=1: 3$ ] 跟踪反应. 反应结束, 滴加稀 $\mathrm{NaOH}$ 溶液中和, 过滤, 用乙醇洗涤产物. 放干后用 DMF-EtOH 重结晶, 得 1-(3- $\beta$-荎基-1-苯基吡唑-4-亚甲 基)-2-(4-芳基噻唑-2-基)-腙类化合物 $\mathbf{4 a} \sim \mathbf{4 g}$.

3.2.5 1-(3- $\beta$ - 菜基-1-苯基吡唑-4-亚甲基)-2-(4-芳基 噻唑-2-基)-腙 $(\mathbf{4 a} \sim 4 \mathrm{~g})$ 的三组分一锅法合成

取 $1 \mathrm{mmol} 3$ - $\beta$-萗基-1-苯基吡唑-4-甲醛和 $1 \mathrm{mmol}$ 氨基硫艮溶于 $10 \mathrm{~mL}$ 无水乙醇中, 在超声波辐射下反 应 $5 \mathrm{~min}$, 然后向此混合溶液中加入 $1 \mathrm{mmol} \omega$-溴代芳基 乙酮, 继续进行超声波辐射反应 $25 \sim 60 \mathrm{~min}$, 用 $\mathrm{TLC}$ $[V$ (甲醇 $): V($ 氯仿 $)=1: 3$ ] 跟踪反应. 反应结束后, 过滤, 用 EtOH 冲洗, 再用 DMF-EtOH 或 DMF- $\mathrm{H}_{2} \mathrm{O}$ 重结晶, 得 1-(3- $\beta$-萗基-1-苯基吡唑-4-亚甲基)-2-(4-芳基噻唑-2基)-腙类化合物 $\mathbf{4 a} \sim \mathbf{4 g}$.

目标化合物 $4 \mathrm{a} \sim 4 \mathrm{~g}$ 的物理数据、核磁共振氢谱、 质谱、红外光谱与元素分析数据如下:

1-(3- $\beta$-萗基-1-苯基吡唑-4-亚甲基)-2-(4-苯基噻唑2-基)-腙(4a): 深砖红色晶体, m.p. $168 \sim 170{ }^{\circ} \mathrm{C} ;{ }^{1} \mathrm{H}$ NMR (DMSO- $\left.d_{6}, 400 \mathrm{MHz}\right) \delta$ : 12.05 (s, $\left.1 \mathrm{H}, \mathrm{NH}\right), 8.96$ (s, $1 \mathrm{H}$, pyrazole-H), $7.30 \sim 8.33(\mathrm{~m}, 18 \mathrm{H}$, Naphthyl- $\mathrm{H}+$ $\mathrm{ArH}+\mathrm{N}=\mathrm{CH}+$ Thiazole-H); ESI-MS $m / z(\%): 494(\mathrm{M}+$ $\left.\mathrm{Na}^{+}, 72\right), 472\left(\mathrm{M}+\mathrm{H}^{+}, 100\right), 298(20)$; IR (KBr) $v: 3370$ $(\mathrm{N}-\mathrm{H}), 3049(\mathrm{Ar}-\mathrm{H}), 1621,1590,1536,1506(\mathrm{C}=\mathrm{C}$, $\mathrm{C}=\mathrm{N}) \mathrm{cm}^{-1}$. Anal. calcd for $\mathrm{C}_{29} \mathrm{H}_{21} \mathrm{~N}_{5} \mathrm{~S}: \mathrm{C} 73.90, \mathrm{H} 4.51$, N 14.90; found C 73.86, H 4.49, N 14.85.
1-(3- $\beta$-萗基-1-苯基吡唑-4-亚甲基)-2-(4-对甲基苯 基噻唑-2-基)-腙(4b): 砖红色晶体, m.p. $170 \sim 172{ }^{\circ} \mathrm{C}$; ${ }^{1} \mathrm{H}$ NMR (DMSO- $\left.d_{6}, 400 \mathrm{MHz}\right) \delta: 12.15$ (s, $\left.1 \mathrm{H}, \mathrm{NH}\right), 8.89$ (s, 1H, Pyrazole-H), $7.19 \sim 8.39$ (m, 18H, Naphthyl-H+ $\mathrm{ArH}+\mathrm{N}=\mathrm{CH}+$ Thiazole-H), $2.5\left(\mathrm{~s}, 3 \mathrm{H}, \mathrm{CH}_{3}\right)$; ESI-MS $m / z(\%): 508\left(\mathrm{M}+\mathrm{Na}^{+}, 100\right), 486\left(\mathrm{M}+\mathrm{H}^{+}, 40\right)$; IR (KBr) $v: 3355(\mathrm{~N}-\mathrm{H}), 3042(\mathrm{Ar}-\mathrm{H}), 1618,1596,1542,1496$ $(\mathrm{C}=\mathrm{C}, \mathrm{C}=\mathrm{N}) \mathrm{cm}^{-1}$. Anal. calcd for $\mathrm{C}_{30} \mathrm{H}_{23} \mathrm{~N}_{5} \mathrm{~S}$ : C 74.24, $\mathrm{H}$ 4.82, N 14.46; found C 74.20, H 4.77, N 14.42 .

1-(3- $\beta$-萗基-1-苯基吡唑-4-亚甲基)-2-(4-对甲氧基 苯基噻唑-2-基)-腙(4c)：砖红色晶体, m.p. $163 \sim 165{ }^{\circ} \mathrm{C}$; ${ }^{1} \mathrm{H}$ NMR (DMSO- $\left.d_{6}, 400 \mathrm{MHz}\right) \delta: 12.14$ (s, 1H, NH), 8.95 (s, 1H, Pyrazole-H), $7.13(\mathrm{~s}, 1 \mathrm{H}, \mathrm{N}=\mathrm{CH}), 6.95 \sim 8.33(\mathrm{~m}$, 16H, Naphthyl-H + ArH + Thiazole-H), 3.83 (s, 3H, $\left.\mathrm{OCH}_{3}\right)$; ESI-MS $m / z(\%): 524 \quad\left(\mathrm{M}+\mathrm{Na}^{+}, 33\right), 502(\mathrm{M}+$ $\left.\mathrm{H}^{+}, 100\right)$; IR (KBr) v: $3362(\mathrm{~N}-\mathrm{H}), 3045(\mathrm{Ar}-\mathrm{H}), 1619$, 1593, 1538, $1504(\mathrm{C}=\mathrm{C}, \mathrm{C}=\mathrm{N}) \mathrm{cm}^{-1}$. Anal. calcd for $\mathrm{C}_{30} \mathrm{H}_{23} \mathrm{~N}_{5} \mathrm{OS}$ : C 71.87, H 4.70, N 13.98; found C 71.84, H 4.62, N 13.96 .

1-(3- $\beta$-荎基-1-苯基吡唑-4-亚甲基)-2-(4-对氯苯基 噻唑-2-基)-腙(4d): 砖红色晶体, m.p. $158 \sim 160{ }^{\circ} \mathrm{C} ;{ }^{1} \mathrm{H}$ NMR (DMSO- $\left.d_{6}, 400 \mathrm{MHz}\right) \delta: 12.003(\mathrm{~s}, 1 \mathrm{H}, \mathrm{NH}), 8.96$ (s, 1H, Pyrazole-H), 7.37 8.32 (m, 18H, Naphthyl-H+ $\mathrm{ArH}+$ Thiazole-H$+\mathrm{N}=\mathrm{CH})$; ESI-MS $m / z(\%): 506(\mathrm{M}+$ $\left.\mathrm{H}^{+}, 33\right), 508\left(\mathrm{M}+2+\mathrm{H}^{+}, 13\right), 528\left(\mathrm{M}+\mathrm{Na}^{+}, 100\right), 529$ $\left(\mathrm{M}+2+\mathrm{Na}^{+}, 34\right), 298$ (12), 274 (14), 242 (14); IR (KBr) $v: 3355(\mathrm{~N}-\mathrm{H}), 3045(\mathrm{Ar}-\mathrm{H}), 1629,1598,1544,1513$ $(\mathrm{C}=\mathrm{C}, \mathrm{C}=\mathrm{N}) \mathrm{cm}^{-1}$. Anal. calcd for $\mathrm{C}_{29} \mathrm{H}_{20} \mathrm{ClN}_{5} \mathrm{~S}: \mathrm{C}$ 68.87, H 4.01, N 13.88; found C 68.83, H 3.98, N 13.84.

1-(3- $\beta$-荎基-1-苯基吡唑-4-亚甲基)-2-(4-对硝基苯 基噻唑-2-基)-腙(4e): 土黄色片状晶体, 产率 $82 \%$. m.p. $247 \sim 249{ }^{\circ} \mathrm{C} ;{ }^{1} \mathrm{H}$ NMR (DMSO- $\left.d_{6}, 400 \mathrm{MHz}\right) \delta$ : 12.12 (s, $1 \mathrm{H}, \mathrm{NH}), 8.97(\mathrm{~s}, 1 \mathrm{H}$, Pyrazole-H), $7.70(\mathrm{~s}, 1 \mathrm{H}, \mathrm{N}=\mathrm{CH})$, $7.55 \sim 8.32(\mathrm{~m}, 17 \mathrm{H}$, Naphthyl-H + Ar-H + Thiazole-H); ESI-MS $m / z(\%): 539\left(\mathrm{M}+\mathrm{Na}^{+}, 100\right), 517\left(\mathrm{M}+\mathrm{H}^{+}, 67\right)$; IR (KBr) v: $3332(\mathrm{NH}), 3031(\mathrm{ArH})$, 1634, 1601, 1555, $1518(\mathrm{C}=\mathrm{C}, \mathrm{C}=\mathrm{N}) \mathrm{cm}^{-1}$. Anal. calcd for $\mathrm{C}_{29} \mathrm{H}_{20} \mathrm{~N}_{6} \mathrm{O}_{2} \mathrm{~S}: \mathrm{C}$ 67.48, H 3.94, N 16.29; found C 67.43, H 3.90, N 16.27.

1-(3- $\beta$-菜基-1-苯基吡唑-4-亚甲基)-2-(4-对溴苯基 噻唑-2-基)-腙(4f): 砖红色晶体, m.p. $169 \sim 171{ }^{\circ} \mathrm{C} ;{ }^{1} \mathrm{H}$ NMR (DMSO- $\left.d_{6}, 400 \mathrm{MHz}\right) \delta$ : 12.04 (s, $\left.1 \mathrm{H}, \mathrm{NH}\right), 8.95$ (s, $1 \mathrm{H}$, Pyrazole-H), $7.38 \sim 8.32(\mathrm{~m}, 18 \mathrm{H}$, Naphthyl-H + $\mathrm{ArH}+$ Thiazole- $\mathrm{H}+\mathrm{N}=\mathrm{CH})$; ESI-MS $m / z(\%): 552(\mathrm{M}+$ $\left.2+\mathrm{H}^{+}, 100\right), 550\left(\mathrm{M}+\mathrm{H}^{+}, 72\right)$; IR $(\mathrm{KBr}) v: 3324(\mathrm{~N}-\mathrm{H})$, $3023(\mathrm{Ar}-\mathrm{H}), 1634,1608,1541,1521(\mathrm{C}=\mathrm{C}, \mathrm{C}=\mathrm{N})$ 
$\mathrm{cm}^{-1}$. Anal. calcd for $\mathrm{C}_{29} \mathrm{H}_{20} \mathrm{BrN}_{5} \mathrm{~S}: \mathrm{C}$ 63.30, H 3.72, N 12.78; found $\mathrm{C} 63.28, \mathrm{H} 3.66$, N 12.72 .

1-(3- $\beta$-菜基-1-苯基吡唑-4-亚甲基)-2-(4- $\beta$-萗基噻 唑-2-基)-腙(4g)：浅黄色晶体, m.p. $159 \sim 161{ }^{\circ} \mathrm{C} ;{ }^{1} \mathrm{H}$ NMR (DMSO- $\left.d_{6}, 400 \mathrm{MHz}\right) \delta$ : 12.1 (s, 1H, NH), 9.02 (s, $1 \mathrm{H}$, Pyrazole-H), $8.34(\mathrm{~s}, 1 \mathrm{H}, \mathrm{N}=\mathrm{CH}), 8.33(\mathrm{~s}, 1 \mathrm{H}$, Naphthyl-H), $7.38 \sim 8.37$ (m, 19H, 2Naphthyl-H+Ar-H+ Thiazole-H); ESI-MS $m / z$ (\%): $544\left(\mathrm{M}+\mathrm{Na}^{+}, 100\right), 522$ $\left(\mathrm{M}+\mathrm{H}^{+}, 18\right), 242(26)$; IR $(\mathrm{KBr}) v: 3391(\mathrm{~N}-\mathrm{H}), 3048$ $(\mathrm{Ar}-\mathrm{H}), 1616,1580,1540,1503(\mathrm{C}=\mathrm{C}, \mathrm{C}=\mathrm{N}) \mathrm{cm}^{-1}$. Anal. calcd for $\mathrm{C}_{33} \mathrm{H}_{23} \mathrm{~N}_{5} \mathrm{~S}$ : C 78.41, H 4.60, N 11.74; found $\mathrm{C} 78.37, \mathrm{H} 4.55, \mathrm{~N} 11.72$.

\section{2 生物活性试验}

为了研究目标化合物对 $\mathrm{H}_{2} \mathrm{O}_{2}$ 诱导 $\mathrm{PC} 12$ 细胞损伤的 影响, 我们参照文献[19]的方法, 建立过氧化氢诱导的 PC12 细胞损伤模型, 用噻唑蓝(MTT) 比色法检测处理 后细胞的生长活力. 简要过程: PC12 细胞接种于 96 孔 板, 每孔 $5 \times 10^{3} \sim 6 \times 10^{3}$ 个, 培养 $24 \mathrm{~h}$ 后观察细胞贴壁 良好则加入含 $100 \mu \mathrm{mol} / \mathrm{L} \mathrm{H}_{2} \mathrm{O}_{2}$ 的完全培养基, 同时加 入一定浓度受试化合物，造模 $24 \mathrm{~h}$ ，向已处理好的 96 孔培养板每孔加入 $0.5 \mathrm{~g} / \mathrm{L}$ MTT $20 \mu \mathrm{L}, 37{ }^{\circ} \mathrm{C}$ 培养 $2 \mathrm{~h}$, 吸弃上清液, 加入 $100 \mu \mathrm{L}$ DMSO 震荡至形成的结晶完 全溶解. 用酶标仪比色, 检测 $570 \mathrm{~nm}$ 处的吸光度值 $(\mathrm{A} 570 \mathrm{~nm})$, 推算细胞存活率.

\section{References}

[1] Sherman, T. D.; Duke, M. V.; Clark, R. D.; Sanders, E. F.; Matsumoto, H.; Duke, O. S. Pestic. Biochem. Phys. 1991, 40, 236.
[2] Karci, F.; Sener, N.; Yamac, M.; Sener, I.; Demircall, A. Dyes Pigm. 2009, 80,47 .

[3] Liu, X. H.; Cui, P.; Song, B. A.; Bhadury, P. S.; Zhu, H. L.; Wang, S. F. Bioorg. Med. Chem. 2008, 16, 4075.

[4] Bekhit, A. A.; Ashour, H. M. A.; AbdelGhany, Y. S.; Bekhit, A. E.; Azza, B. Eur. J. Med. Chem. 2008, 43, 456.

[5] Abdel-Aziz, M.; Farag, H. Bioorg. Med. Chem. 2009, 17, 3829.

[6] Fan, Z.-J.; Zhong, B.; Wang, S.-H. Chin. J. Appl. Chem. 2003, 20, 365 (in Chinese).

(范志金, 钟滨, 王素华, 应用化学, 2003, 20, 365.)

[7] Rollas, S.; Gülerman, N.; Erdeniz, H. Farmaco 2002, 57, 171.

[8] Salem, A. A. Microchem. J. 1998, 60, 51.

[9] Poon, S. F.; Jean, S. J. D.; Harrington, P. E.; Henley, C.; Davis, J.; Morony, S.; Lott, F. D.; Reagan, J. D.; Lu, J. Y.; Yang, Y.; Fotsch, C. J. Med. Chem. 2009, 52, 6535 .

[10] Li, D.-M.; Lv, L.-F.; Sun, P.-P.; Zhou, W.; Wang, P.; Wu, J.-Y.; Kan, Y.-H.; Zhou, H.-P.; Tian, Y.-P. Dyes Pigm. 2009, 83, 180.

[11] Manzano, B. R.; Jalón, F. A.; Espino, G.; Guerrero, A.; Claramunt, R. M.; Escolástico, C.; Elguero, J.; Heras, M. A. Polyhedron 2007, 26, 4373.

[12] Baraldi, P. G.; Tabrizi, M. A.; Preti, D.; Bovero, A.; Fruttarolo, F.; Romagnoli, R.; Zaid, N. A.; Moorman, A. R.; Varani, K.; Borea, P. A. J. Med. Chem. 2005, 48, 5001.

[13] Chimenti, F.; Bizzarri, B.; Bolasco, A.; Secci, D.; Chimenti, P.; Granese, A.; Carradori, S.; D'Ascenzio, M.; Lilli, D.; Rivanera, D. Eur. J. Med. Chem. 2011, 46, 378.

[14] Secci, D.; Bizzarri, B.; Bolasco, A.; Carradori, S.; D'Ascenzio, M.; Rivanera, D.; Mari, E.; Polletta, L.; Zicari, A. Eur. J. Med. Chem. 2012, 53, 246.

[15] Zhang, D.-N.; Li, J.-T.; Song, Y.-L.; Liu, H.-M.; Li, H,-Y. Ultrson. Sonochem. 2012, 19, 475.

[16] Henry A. Torrey, C. M. Brewster J. Am. Chem. Soc. 1909, 31, 1322.

[17] Hill, G. A.; Kropa, L. E. J. Am. Chem. Soc. 1993, 55, 2509.

[18] Bernard, M.; Hulley, E.; Molenda, H. Stochla, K.; Wrzeciono, U. Pharmazie 1986, 41, 560.

[19] Ablajan, K. Chin. J. Org. Chem. 2011, 31, 724 (in Chinese). (阿布拉江·克依木, 有机化学, 2011, 31, 724.) 